\title{
A Trefftz approach for medium-frequency vibrations of orthotropic
}

\section{structures}

\author{
Louis Kovalevsky ${ }^{\mathrm{a}}$, Hervé Riou ${ }^{\mathrm{b}}$, Pierre Ladevèze ${ }^{\mathrm{b}, *}$ \\ ${ }^{a}$ University of Cambridge, Engineering Department, Dynamics and Vibration Research Group, Trumpington Street, Cambridge CB21PZ, United Kingdom \\ ${ }^{\mathrm{b}}$ LMT-Cachan (ENS Cachan/CNRS/Paris 6 University, PRES UniverSud Paris), 61 avenue du Président Wilson, F-94230 Cachan, France
}

\begin{abstract}
The Variational Theory of Complex Rays (VTCR) was developed in order to calculate the vibrational response of structures in the medium-frequency range. It leads to a numerical approximation of this response through the resolution of a small system of equations which, contrary to element-based methods, does not result from a refined spatial discretization. This strategy has already been validated for assemblies of isotropic plates. In this paper, we extend it to the case of orthotropic planar structure. The method is illustrated through various orthotropic plate vibration problems under several types of harmonic loading in the medium-frequency range.
\end{abstract}

\section{Introduction}

Undoubtedly, the modeling and analysis of the vibrational response of structures are among the key issues in the design of many industrial systems. Today, even for complex structures, there remain no major difficulties in the low-frequency range, at least concerning modeling and analysis (see [1]). However, the modeling and analysis of medium-frequency vibrations, on which this paper focuses, continue to cause problems. Attempting to extend lowfrequency methods to this case is difficult because the length of variation of the phenomena being studied is very small compared to the characteristic dimension of the structure and, therefore, the finite element calculation would require a prohibitive number of degrees of freedom. This difficulty has led to the development of a number of improved finite element approaches (see [2-11]).

Today, there are also dedicated computational strategies for the resolution of medium-frequency problems, known as Trefftz methods [12], which differ from element-based methods in that they use shape functions which are exact solutions of the governing differential equations. These approaches include, for example, a special use of the partition of unity method $[13,14]$, the ultra-weak variational method [15-17], the least-squares method [18,17], the discontinuous enrichment method [19-21], the wave boundary element method [22,23], and the wave-based

\footnotetext{
* Corresponding author. Tel.: +33 147402238; fax: +33 147402240.

E-mail addresses: louis.kovalevsky@eng.cam.ac.uk (L. Kovalevsky), riou@lmt. ens-cachan.fr (H. Riou), ladeveze@lmt.ens-cachan.fr (P. Ladevèze).
}

method [24-26]. All these numerical techniques undertake to solve the problem using oscillating functions, the main differences being in the treatment of the transmission conditions between substructures and the boundary conditions. The Variational Theory of Complex Rays (VTCR), which is the subject of this paper, is one of these dedicated Trefftz methods.

The VTCR is a predictive tool specifically designed to deal with medium-frequency problems which was introduced in [27]. Its first characteristic is that it uses a special weak formulation of the problem which makes the approximations within the substructures a priori independent of one another. Thus, any type of shape function can be used in a substructure provided that it satisfies the governing equations. This gives the approach great flexibility and, consequently, makes it very efficient because any type of approximation function can be used without any difficulty. The second characteristic is the introduction of an approximation with a strong mechanical content: the solution is described as the superposition of an infinite number of plane waves which satisfy the governing equations exactly. All the wave directions are taken into account and their amplitudes are the unknowns of the problem.

Previous works have already validated this strategy for 2D assemblies of isotropic plates (see [28]) and for 3D isotropic assemblies (see [29]), but many industrial structures contain composite materials which are not isotropic. Therefore, this paper goes one step further in the development of the VTCR by enabling the designer to take the properties of these materials into account. However, we will assume that the composite material can be described as an orthotropic material. The paper is structured as 
follows: Section 2 presents the reference problem and its solution using the VTCR; Section 3 illustrates the strategy in the case of a vibrating orthotropic plate; finally, conclusions are drawn in Section 4.

\section{Basic aspects of the VTCR}

\subsection{The reference problem}

The reference problem being considered is the steady-state vibration of an assembly of plates at a fixed circular frequency $\omega$. In order to simplify the presentation, let us assume that the structure consists of two coplanar plates, but this can be easily generalized to an assembly of any number of plates of any geometry. These plates are assumed to be thin, homogeneous, orthotropic and governed by the elastic Kirchhoff-Love theory. We are to study the steady-state vibrations of such an assembly. Classically, all quantities are defined as complex quantities, i.e. an amplitude $Q(\mathbf{x})$ is associated with $Q(\mathbf{x}) e^{i \omega t}$. Let $S_{1}$ and $S_{2}$ denote the mean surfaces of the two plates and $\partial S_{1}$ and $\partial S_{2}$ their boundaries. Their thickness, stiffness tensor (including damping) and density are designated respectively by $h_{e}, \mathbf{D}_{e}$ and $\rho_{e}, e \in\{1,2\}$. The boundary conditions are a prescribed deflection $w_{e}^{d}$ along $\partial_{w} S_{e}$, a prescribed slope $w_{n e}^{d}$ along $\partial_{w n} S_{e}$, a bending moment $M_{e}^{d}$ along $\partial_{M} S_{e}$ and a shear force $K_{e}^{d}$ along $\partial_{K} S_{e}$, with $e \in\{1,2\}$. The quantities of interest are the displacements $w_{e}$ and the moment operators $\mathbf{M}_{e}$.

The reference problem is: find $\left(w_{e}, \mathbf{M}_{e}\right) \in \mathcal{W} \times \mathcal{M}$ (set of the finite-energy fields) such that:

$$
\begin{aligned}
& \mid \begin{array}{ll}
\operatorname{div}\left(\operatorname{div} \mathbf{M}_{e}\right)+p_{e}=-\rho_{e} h_{e} \omega^{2} w_{e} & \text { in } S_{e}, e \in\{1,2\} \\
\mathbf{M}_{e}=\mathbf{D}_{e} \mathbf{X}\left(w_{e}\right) & \text { in } S_{e}, e \in\{1,2\}
\end{array} \\
& \mid \begin{array}{ll}
w_{e}=w_{e}^{d} & \text { along } \partial_{w} S_{e}, e \in\{1,2\} \\
w_{e, n_{e}}=w_{n e}^{d} & \text { along } \partial_{n w} S_{e}, e \in\{1,2\} \\
\mathbf{n}_{e} \mathbf{M}_{e} \mathbf{n}_{e}=M_{e}^{d} & \text { along } \partial_{M} S_{e}, e \in\{1,2\} \\
K_{e}=\mathbf{n}_{e} \operatorname{div}\left(\mathbf{M}_{e}\right)+\left(\mathbf{n}_{e} \mathbf{M}_{e} \mathbf{n}_{e}\right)_{, t}=K_{e}^{d} & \text { along } \partial_{K} S_{e}, e \in\{1,2\} \\
\left.\left[\mathbf{t}_{e} \mathbf{M}_{e} \mathbf{n}_{e}\right]\right]=0 & \text { at the corner of } \partial S_{e}, e \in\{1,2\} \\
w_{1}=w_{2} & \text { along } \partial S_{1} \cap \partial S_{2} \\
w_{1, n_{1}}=w_{2, n_{2}} & \text { along } \partial S_{1} \cap \partial S_{2} \\
\mathbf{n}_{1} \mathbf{M}_{1} \mathbf{n}_{1}+\mathbf{n}_{2} \mathbf{M}_{2} \mathbf{n}_{2}=0 & \text { along } \partial S_{1} \cap \partial S_{2} \\
K_{1}+K_{2}=0 & \text { along } \partial S_{1} \cap \partial S_{2}
\end{array}
\end{aligned}
$$

where $p_{e}$ is a prescribed pressure applied over $S_{e}, \mathbf{n}_{1}$ the outward normal to $S_{e}$ and $\mathbf{t}_{e}$ the tangent to $\partial S_{e}$. [[ $\left.\left.\square\right]\right]$ denotes the value of the jump of quantity $\square$ and $\mathbf{X}$ is the curvature operator. If $\mathbf{D}_{e}$ are positive definite and the damping factors $\eta_{e}$ of each plate are positive, the solution of the reference problem is unique.

\subsection{The variational formulation associated with the VTCR}

The VTCR uses a global formulation of Problem (1) and (2) in terms of both displacements and forces along with the boundary conditions and transmission conditions. First, one must define $\mathcal{S}_{e}^{a d}$, the functional space that satisfies the governing Eqs. (1):

$$
\left(w_{e}, \mathbf{M}_{e}\right) \in \mathcal{S}_{e}^{a d} \Longleftrightarrow \mid \begin{aligned}
& \left(w_{e}, \mathbf{M}_{e}\right) \in \mathcal{W} \times \mathcal{M} \\
& \operatorname{div}\left(\mathbf{d i v}_{\mathbf{e}}\right)+p_{e}=-\rho_{e} h_{e} \omega^{2} w_{e} \\
& \mathbf{M}_{e}=\mathbf{D}_{e} \mathbf{X}\left(w_{e}\right)
\end{aligned}
$$

Similarly, let $\mathcal{S}_{e, 0}^{a d}$ be the functional space which satisfies the homogeneous governing equations (the same equations as (3), but without $p_{e}$ ). Then, the VTCR formulation of Problem (1) and (2) is: find $\left(w_{1}, \mathbf{M}_{1}\right) \in \mathcal{S}_{1}^{a d}$ and $\left(w_{2}, \mathbf{M}_{2}\right) \in \mathcal{S}_{2}^{a d}$ such that:

$$
\begin{aligned}
& \int_{\partial w_{w_{W}} S_{1}} \mathbf{n}_{1} \delta \mathbf{M}_{1} \mathbf{n}_{1}\left(w_{1, n_{1}}-w_{n 1}^{d}\right)^{*} \mathrm{~d} \partial S_{1}-\int_{\partial_{w} S_{1}} \delta K_{1}\left(w_{1}-w_{1}^{d}\right)^{*} \mathrm{~d} \partial S_{1} \\
& +\int_{\partial_{M} S_{1}}\left(\mathbf{n}_{1} \mathbf{M}_{1} \mathbf{n}_{1}-M_{1}^{d}\right) \delta w_{1, n_{1}}^{*} \mathrm{~d} \partial S_{1}-\int_{\partial_{K} S_{1}}\left(K_{1}-K_{1}^{d}\right) \delta w_{1}^{*} \mathrm{~d} \partial S_{1} \\
& +\int_{\partial_{W_{w n} S_{2}}} \mathbf{n}_{2} \delta \mathbf{M}_{2} \mathbf{n}_{2}\left(w_{2, n_{2}}-w_{n 2}^{d}\right)^{*} \mathrm{~d} \partial S_{2}-\int_{\partial_{w} S_{2}} \delta K_{2}\left(w_{2}-w_{2}^{d}\right)^{*} \mathrm{~d} \partial S_{2} \\
& +\int_{\partial_{M} S_{2}}\left(\mathbf{n}_{2} \mathbf{M}_{2} \mathbf{n}_{2}-M_{2}^{d}\right) \delta w_{2, n_{2}}^{*} \mathrm{~d} \partial S_{2}-\int_{\partial_{K} S_{2}}\left(K_{2}-K_{2}^{d}\right) \delta w_{2}^{*} \mathrm{~d} \partial S_{2} \\
& \left.-\sum_{\partial S_{1} \text { corners }}\left[\left[\mathbf{t}_{1} \mathbf{M}_{1} \mathbf{n}_{1}\right]\right] \delta w_{1}^{*}-\sum_{\partial S_{2} \operatorname{corners}}\left[\mathbf{t}_{2} \mathbf{M}_{2} \mathbf{n}_{2}\right]\right] \delta w_{2}^{*} \\
& +\int_{\partial S_{1} \cap \partial S_{2}} \frac{1}{2}\left[\delta\left(\mathbf{n}_{1} \mathbf{M}_{1} \mathbf{n}_{1}-\mathbf{n}_{2} \mathbf{M}_{2} \mathbf{n}_{2}\right)\left(w_{1, n_{1}}-w_{2, n_{2}}\right)^{*}-\delta\left(K_{1}-K_{2}\right)\left(w_{1}-w_{2}\right)^{*}\right] \mathrm{d} \Gamma \\
& +\int_{\partial S_{1} \cap \partial S_{2}} \frac{1}{2}\left[\left(\mathbf{n}_{1} \mathbf{M}_{1} \mathbf{n}_{1}+\mathbf{n}_{2} \mathbf{M}_{2} \mathbf{n}_{2}\right) \delta\left(w_{1, n_{1}}+w_{2, n_{2}}\right)^{*}-\left(K_{1}+K_{2}\right) \delta\left(w_{1}+w_{2}\right)^{*}\right] \mathrm{d} \Gamma=0 \\
& \forall\left(\delta w_{1}, \delta \mathbf{M}_{1}\right) \in \mathcal{S}_{1,0}^{a d}, \forall\left(\delta w_{2}, \delta \mathbf{M}_{2}\right) \in \mathcal{S}_{2,0}^{a d}
\end{aligned}
$$

where $\square^{*}$ denotes the complex conjugate of $\square$. (4) can be shortened to: find $s=(w, \mathbf{M}) \in \mathcal{S}^{a d}$ such that:

$a(s, \delta s)=l(\delta s) \quad \forall \delta s \in \mathcal{S}_{0}^{a d}$

In order to find an approximate solution of (1), (2), the basic idea is to seek a solution of (5) in a space $\mathcal{S}^{\text {had }} \subset \mathcal{S}^{\text {ad }}$ (and, consequently, $\left.\mathcal{S}_{0}^{h, a d} \subset \mathcal{S}_{0}^{a d}\right)$ of finite dimension: find $s^{h}=\left(w^{h}, \mathbf{M}^{h}\right) \in \mathcal{S}^{h, a d}$ such that:

$a\left(s^{h}, \delta s^{h}\right)=l\left(\delta s^{h}\right) \quad \forall \delta s^{h} \in \mathcal{S}_{0}^{h, a d}$

\subsection{Construction of admissible fields}

In order to define the $\mathcal{S}^{\text {h,ad }}$ term in (6), we must find the exact solutions of the governing Eqs. (1). In the case of orthotropic plates, assuming that the principal directions of orthotropy coincide with the $x$ and $y$ coordinates of the plate's mean surface, (1) leads to:

$D_{e x} \frac{\partial^{4} w_{e}}{\partial x^{4}}+2 H_{e} \frac{\partial^{4} w_{e}}{\partial x^{2} \partial y^{2}}+D_{e y} \frac{\partial^{4} w_{e}}{\partial y^{4}}-\rho_{e} h_{e} \omega^{2} w_{e}=p_{e}$

where $D_{e x}=\frac{E_{e x}}{1-v_{e x} v_{e y}} \frac{h_{e}^{3}}{12}, D_{e y}=\frac{E_{e y}}{1-v_{e x} v_{e y}} \frac{h_{e}^{3}}{12}$ and $H_{e}=D_{e x y}+2 D_{e s}$ with $D_{e x y}=\frac{E_{e x} v_{e y}}{1-v_{e x} v_{e y}} \frac{h_{e}^{3}}{12}$ and $D_{e s}=G_{e} \frac{h_{e}^{3}}{12}$, and where $E_{e x}, E_{e y}, v_{e x}, v_{e y}$ and $G_{e}$ are the classical elastic constants of the orthotropic material of plate $S_{e}$ (see [30]). Absorption in the plate is represented through damping factors $\eta_{e x}, \eta_{e y}$ and $\eta_{e}: E_{e x}=\left(1+i \eta_{e x}\right) E_{e x 0}, E_{e y}=\left(1+i \eta_{e y}\right) E_{e y 0}$ and $G_{e}=\left(1+i \eta_{e}\right) G_{e 0}$.

In each substructure $\Omega_{e}, w_{e}$ is sought in the form $w_{e}=w_{e}^{0}+w_{e}^{p}$, where $w_{e}^{p}$ is a known particular solution of (7) while $w_{e}^{0} \in S_{a d, 0}^{e}$ is taken as a sum of Herglotz wave functions, i.e. an integral superposition of propagative or evanescent plane waves of the form:

$w_{e}^{0}(\mathbf{x}):=\int_{\mathcal{C}} A_{e}^{p}(\theta) e^{i \mathbf{k}_{e}^{p}(\theta) \cdot\left(\mathbf{x}-\mathbf{x}_{e}^{p}\right)} \mathrm{d} \theta+\sum_{b} \int_{\mathcal{C}} A_{e}^{b}(\theta) e^{i \mathbf{k}_{e}^{b}(\theta) \cdot\left(\mathbf{x}-\mathbf{x}_{e}^{b}\right)} \mathrm{d} \theta$.

Any Herglotz wave function is a solution of homogeneous Eq. (7). Keeping the terminology from previous works on the VCTR, functions $A_{e}^{p}$ and $A_{e}^{b}$ are called the amplitude portrait of $w_{e}$.

In (8), $\mathcal{C}$ is the unit circle, $\mathbf{x}_{e}^{p}$ is a reference point in $\Omega_{e}, \mathbf{x}_{e}^{b}$ is a reference point along edge $b, A_{e}^{p}(\theta)$ is the amplitude of the propagative plane wave in direction $\theta$ which contributes to $w_{e}$, and $A_{e}^{b}(\theta)$ is the amplitude of the evanescent plane wave in direction $\theta$ which contributes to $w_{e}$. The wave vectors $\mathbf{k}_{e}^{p}(\theta)$ and $\mathbf{k}_{e}^{b}(\theta)$ are defined by:

$\begin{aligned} \mathbf{k}_{e}^{p}(\theta) & =k_{x e} \cos (\theta) \mathbf{e}_{x}+k_{y e} \sin (\theta) \mathbf{e}_{y} \\ \mathbf{k}_{e}^{b}(\theta) & =-i k_{n e} \sqrt{1+\cos ^{2}(\theta / 2)} \mathbf{n}_{b}+k_{t e} \cos (\theta / 2) \mathbf{t}_{b}\end{aligned}$ 
where $k_{x e}=\left(\frac{\rho h \omega^{2}}{D_{e x}}\right)^{1 / 4}, k_{y e}=\left(\frac{\rho h \omega^{2}}{D_{e y}}\right)^{1 / 4}, \mathbf{e}_{x}$ and $\mathbf{e}_{y}$ are the directions of orthotropy of plate $\Omega_{e}$, and $\mathbf{n}_{b}$ and $\mathbf{t}_{b}$ are respectively the outward normal and the tangent of edge $b$. The values of $k_{n}$ and $k_{t}$ are determined by the equations:

$\mathbf{k}_{n e}=k_{x e} \mathbf{e}_{x} \cdot \mathbf{n}_{b}+k_{y e} \mathbf{e}_{y} \cdot \mathbf{n}_{b} \quad \mathbf{k}_{t e}=k_{x e} \mathbf{e}_{x} \cdot \mathbf{t}_{b}+k_{y e} \mathbf{e}_{y} \cdot \mathbf{t}_{b}$

In order to discretize and get the VCTR formulation (6), each amplitude portrait $A_{e}^{\square}$ (where $\square$ is either $p$ or $b$ ) is discretized as a sum of $N_{e}^{\square}$ Dirac distributions supported at angular locations $\theta_{n}$. Then, $w_{e}^{h}$ is the superposition of a finite number of plane waves:

$w_{e}^{h}(\mathbf{x}):=w_{e}^{p}+\sum_{n=1}^{N_{e}^{p}} a_{n}^{p} e^{i \mathbf{k}_{e}^{p}\left(\theta_{n}^{p}\right) \cdot\left(\mathbf{x}-\mathbf{x}_{e}^{p}\right)}+\sum_{b} \sum_{n=1}^{N_{e}^{b}} a_{n}^{b} e^{i \mathbf{k}_{e}^{b}\left(\theta_{n}^{b}\right) \cdot\left(\mathbf{x}-\mathbf{x}_{e}^{b}\right)}$

where $a_{n}^{\square}$ are the unknowns of the problem and $\theta_{n}^{\square}=n \frac{2 \pi}{N_{e}^{\square}}$.

The discretization parameter $N_{e}^{\square}$ (and, therefore, $\operatorname{Dim}\left(\mathcal{S}_{e, 0}^{h, a d}\right)$ ) can be set a priori using a heuristic geometrical criterion [31] which says that it is proportional to the ratio of the characteristic dimension $R_{e}^{\square}$ of the domain $\Omega_{e}$ (for propagative waves) or the edge $b$ (for evanescent waves) to the wavelength:

$N_{e}^{\square}=\left[\alpha \frac{k R_{e}^{\square}}{2 \pi}\right]$

where $\alpha$ is a proportionality coefficient, $k$ the biggest value between $k_{x e}$ and $k_{y e}$, and the brackets denote the integer part.

\subsection{The discretized forme of the VTCR}

With each plate $\Omega_{e}$ discretized according to (12), the VTCR formulation (6) leads to a linear system of equations in the complex domain:

$\mathbf{A a}=\mathbf{b}$

Matrix A corresponds to the discretization of the bilinear form of weak formulation (6), $\mathbf{a}$ is the vector of the unknown quantities which approximate the distribution of the wave amplitudes and $\mathbf{b}$ corresponds to the discretization of the linear form of (6). The integrations that we have to do in the variational formulation (6) have an analytical expression for straight edge. As a consequence, if the edge of the domain $S$ can be decomposed in straight lines, the assembling process of the VTCR is not computationally costly. If the edges are curved, a first strategy is to decompose each edge into straight segments. However if the radius of curvature is small, a numerical integration has to be done, but it is restricted to the very few curved edges, which does not impair the computational efficiency of the VTCR.

Like any other Trefftz method, the obtained linear system could be badly conditioned. Therefore, one has to avoid the use of a direct solver. One solution is to calculate a low order pseudo inverse, but it requires the singular value decomposition of the matrix A. An other solution, is to use an LSQR iterative solver. In practice the two methods give similar result, with an advantage for the iterative solver in terms of speed. In this next section, the numerical results are obtain using a LSQR. As soon as a is known, the VTCR approximate solution of Problem (1) and (2) is given by Eq. (12).

\section{Numerical results}

The purpose of this section is to assess the performance of the VTCR in the resolution of composite plate vibration problems. In order to do that, let us consider the following two problems: (a) a plane wave traveling through a composite plate and (b) the vibration of a plate under a point force. In both cases, the exact solution is known. The convergence can be measured using the relative error:

$\varepsilon=\frac{\int_{\Omega}\left|w-w^{e x}\right| \mathrm{d} x}{\int_{\Omega}\left|w^{e x}\right| \mathrm{d} x}$

where $w$ is the calculated solution and $w^{e x}$ the exact solution.

In the first example, the plate is modeled using one single domain for the VTCR, whereas in the second example the plate is modeled using either 1, 4 or 8 sub-domains. The number of DOFs of the VTCR is simply the sum of the number of waves used in each element. Let us recall that one could reduce this number by using condensation, but we did not do that in order to make a fair comparison.

\subsection{The case of a plane wave traveling through a composite plate}

Let us consider a plane wave traveling through a rectangular plate $S$ of dimensions $3 \mathrm{~m} \times 2 \mathrm{~m}$. The principal directions of orthotropy are parallel to the sides and the material properties are $E_{x 0}=125 \mathrm{GPa}, E_{y 0}=60 \mathrm{GPa}, \eta_{x}=0.001, \eta_{y}=0.005, v_{x}=0.3$, $v_{y}=0.144$ and $G=72 \mathrm{GPa}$. The density is $\rho=700 \mathrm{~kg} / \mathrm{m}^{3}$ and the thickness is $h=0.01 \mathrm{~m}$. The boundary conditions in terms of the prescribed deflection and slope are such that the exact solution is a plane wave traveling in the direction $\varphi=\frac{2340}{8001} \pi$. This particular direction was chosen because it does not coincide with any of the shape functions used. In this example, one should remark that one has to solve the homogeneous Eq. (1) or (7) (i.e. the term $p_{e}$ is zero) and therefore there is no particular solution.

We considered three circular frequencies: $\omega=10,000 \mathrm{rad} / \mathrm{s}$, $\omega=70,000 \mathrm{rad} / \mathrm{s}$ and $\omega=130,000 \mathrm{rad} / \mathrm{s}$. Fig. 1 shows the exact solution of the problem for each frequency.

Fig. 2 shows the evolution of Error (15) as a function of the number of degree of freedom. The number of shape functions was set using Eq. (13) with $\alpha$ varying from 0.1 to 3 . One can see that at all the frequencies considered the VTCR led to an error of $10^{-5}$ or less, which shows its efficiency on that kind of problem.

\subsection{Vibrations of a composite plate subjected to a harmonic point load}

The second example also concerns a rectangular plate, of dimensions $\left[0, L_{x}\right] \times\left[0, L_{y}\right]$, with $L_{x}=3 \mathrm{~m}$ and $L_{y}=2 \mathrm{~m}$, which is simply supported along all four edges. The principal directions of orthotropy are parallel to the sides. For this problem, the material properties are $E_{x 0}=125 \mathrm{GPa}, E_{y 0}=60 \mathrm{GPa}, v_{x}=0.3, v_{y}=0.144$, $G=70 \mathrm{GPa}, \rho=700 \mathrm{~kg} \mathrm{~m}^{-3}, h=0.01 \mathrm{~m}$ and $\eta_{x}=\eta_{y}=0.0001$. This plate is subjected to a harmonic point load of intensity $F=1000 \mathrm{~N}$ applied at $x_{F}=1.2 \mathrm{~m}$ and $y_{F}=0.7 \mathrm{~m}$. The circular frequencies considered are $\omega=2 \pi \times 100 \mathrm{rad} \mathrm{s}^{-1}, 2 \pi \times 500 \mathrm{rad} \mathrm{s}^{-1}$ and $2 \pi \times$ $900 \mathrm{rad} \mathrm{s}^{-1}$.

The exact solution of this problem can be calculated using Navier's method:

$$
\begin{aligned}
w^{e x}(x, y, \omega)= & \sum_{m=1}^{\infty} \sum_{n=1}^{\infty} \frac{4 F /\left(L_{x} L_{y}\right) \sin \left(m \pi x_{F} / L_{x}\right) \sin \left(n \pi y_{F} / L_{y}\right)}{\rho h\left(\omega_{m n}^{2}-\omega^{2}\right)} \\
& \times \sin \left(m \pi x / L_{x}\right) \sin \left(n \pi y / L_{y}\right)
\end{aligned}
$$

with $\quad \omega_{m n}^{2}=\frac{1}{\rho h}\left(D_{x} m^{4} \pi^{4} L_{x}^{-4}+2 H m^{2} n^{2} \pi^{4} L_{x}^{-2} L_{y}^{-2}+D_{y} n^{4} \pi^{4} L_{y}^{-4}\right)$. The solutions corresponding to the three frequencies considered are shown in Fig. 3.

Using the VTCR, the displacement field was approximated by using Eq. (12), in which the particular solution $w^{p}$ was obtained using the Green function. The converged VTCR solutions when using one single domain with $\alpha=2$ in (13) are shown in Fig. 4. One can see that these solutions are similar to the exact solutions of Fig. 3: all the vibration peaks coincide and the magnitudes of the displacements are the same. 

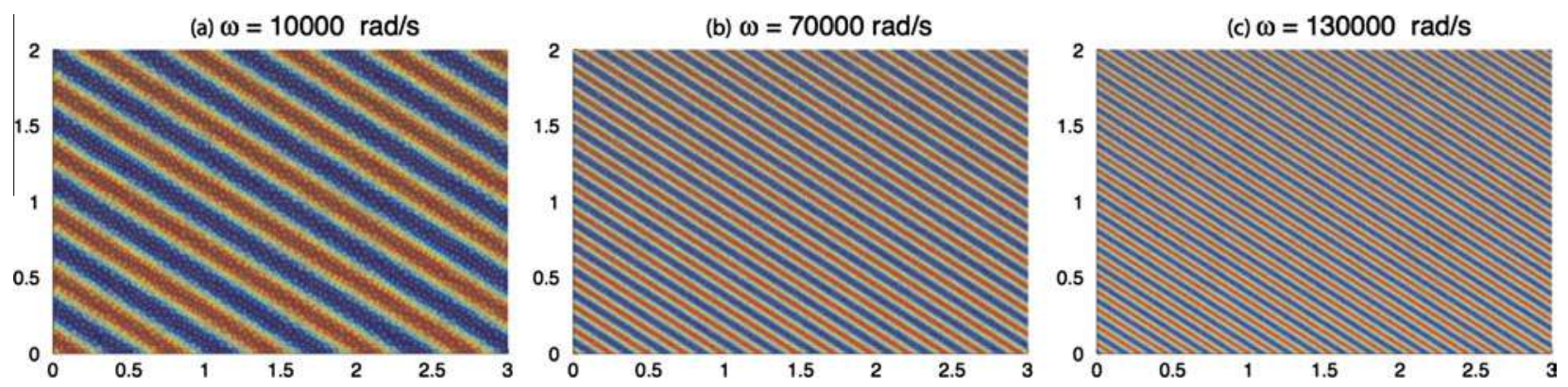

Fig. 1. The exact solution of the orthotropic plate vibration problem of Section 3.1 for $\omega=10,000 \mathrm{rad} / \mathrm{s}(\mathrm{a}), \omega=70,000 \mathrm{rad} / \mathrm{s}(\mathrm{b}) \mathrm{and} \omega=130,000 \mathrm{rad} / \mathrm{s}(\mathrm{c})$.

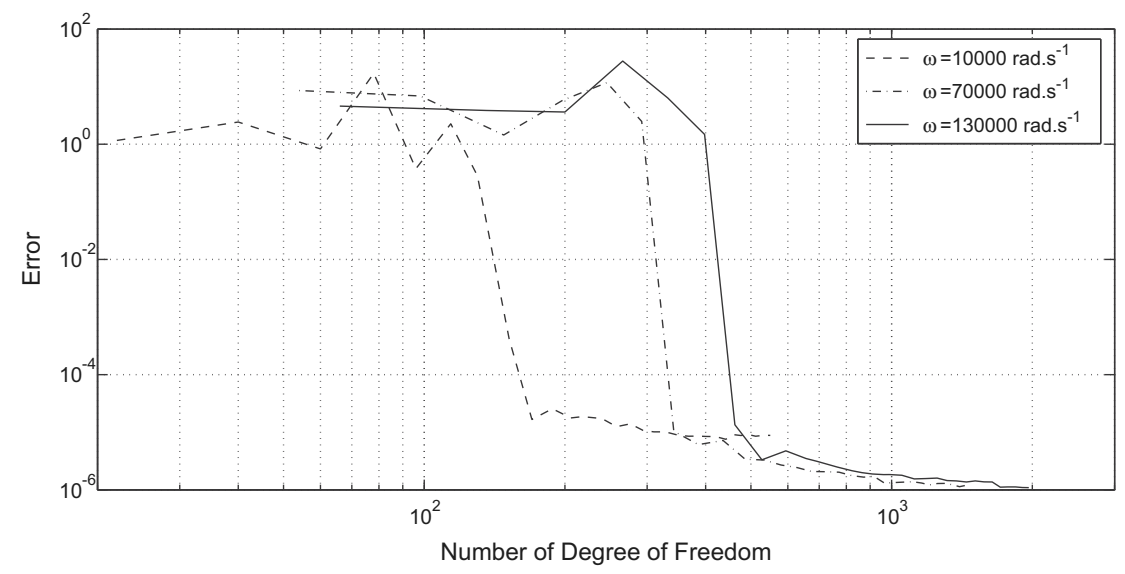

Fig. 2. Performance of the VTCR for the orthotropic plate vibration problem of Section 3.1.

(a)
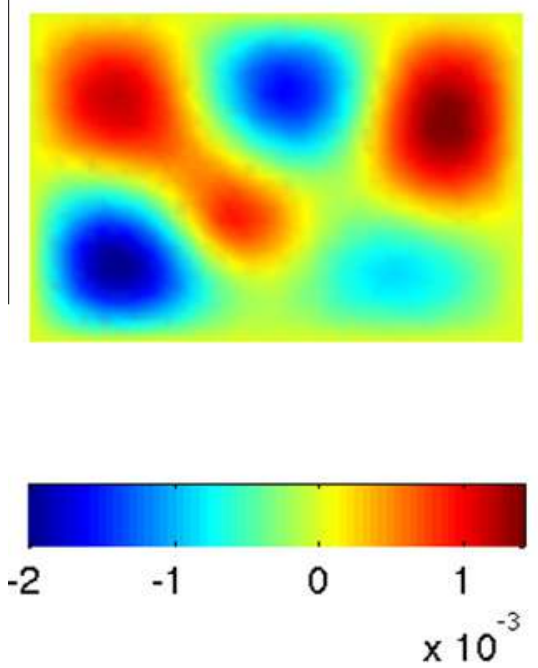

(b)
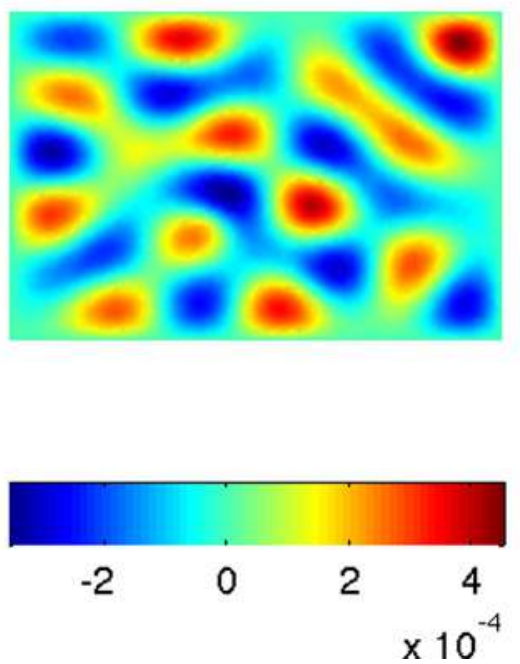

(c)
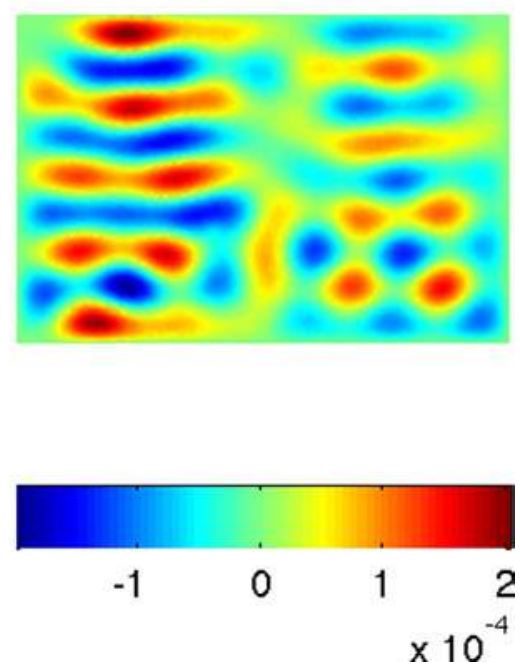

Fig. 3. The exact solutions of the orthotropic plate vibration problem of Section 3.2 for $\omega=2 \pi \times 100 \mathrm{rad} \mathrm{s}^{-1}$ (a), $2 \pi \times 500 \mathrm{rad} \mathrm{s}^{-1}$ (b) and $2 \pi \times 900 \mathrm{rad} \mathrm{s}^{-1}$ (c).

In order to assess the performance of the VTCR, let us compare these results to those obtained by the finite element method using Kirchhoff quadrilateral elements [32]. Fig. 5 shows the FEM and VTCR errors (15) as functions of the number of DOFs for each solution and at each frequency. The VTCR values were obtained using Criterion (13) with $\alpha$ varying from 0.1 to 5 using either 1,4 or 8 identical sub-domains. The FEM values were obtained using meshes with 3-18 Kirchhoff quadrilateral elements per wavelength.
One can see that when using one single sub-domain the performance of the VTCR is significantly better than that of the FEM. At the three frequencies considered, the VTCR converged using considerably fewer DOFs than the FEM (about 100 times fewer for the same error at all the frequencies considered). However, one could also remark than the VTCR is more efficient when taking fewer sub-domains.

One can observe that the frequencies considered were relatively low compared to the first example. In this example, the frequency 
(a)
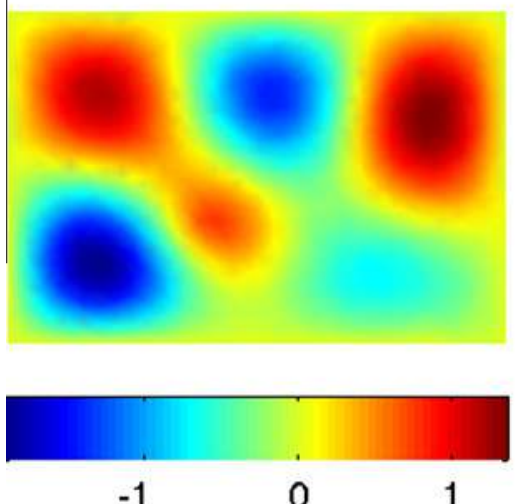

$-1$ (b)
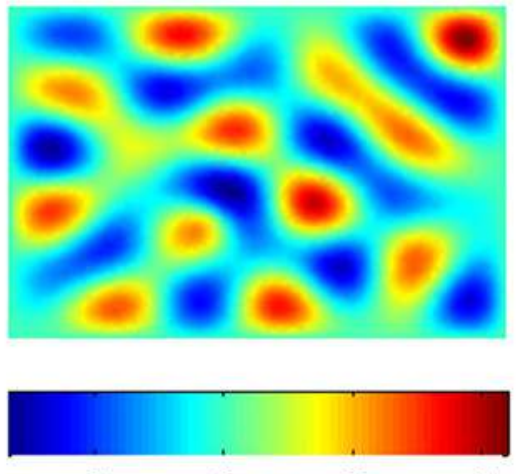

$-2$
0
2 (c)
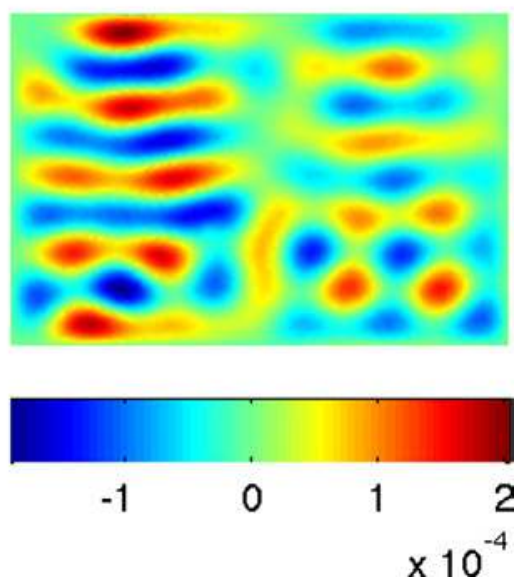

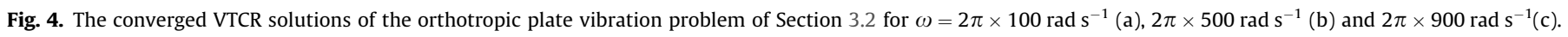

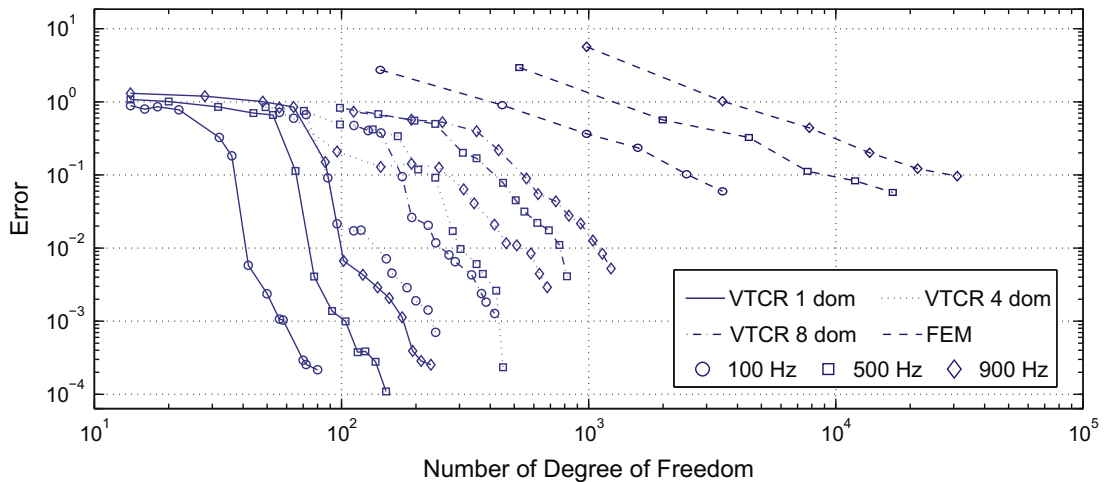

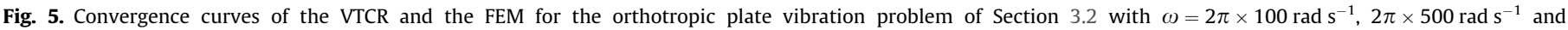
$2 \pi \times 900 \mathrm{rad} \mathrm{s}^{-1}$.

limit was set by the finite element calculation: at higher frequencies, the FE problem would have been too large for a standard desktop computer.

\section{Conclusion}

The prediction of the vibrational behavior of planar orthotropic structures (such as composite panel) is needed in many industrial applications. Harmonic analysis in the medium-frequency range using the standard FEM is difficult because it requires a highly refined mesh. This paper introduced a means of predicting this behavior through the VTCR using plane waves as shape functions. The efficiency of this strategy had already been proven for similar problems involving isotropic plate assemblies. Here, we extended it to orthotropic plates. The numerical tests performed on two different sample problems show that the VTCR leads to accurate numerical solutions using far fewer DOFs than the standard FEM. Our future works will concern the extension of the VTCR to vibro-acoustic coupling.

\section{References}

[1] Craig Jr R. Substructure methods in vibration. J Mech Des 1995:117:207.

[2] Soize C. Reduced models in the medium frequency range for the general dissipative structural dynamic systems. Euro J Mech - A/Solids 1998; 17:657-85.

[3] Harari I, Hughes T. Galerkin/least-squares finite element methods for the reduced wave equation with non-reflecting boundary conditions in unbounded domains. Comput Methods Appl Mech Eng 1992;98(3):411-54.
[4] Babuška I, Ihlenburg F, Paik E, Sauter S. A generalized finite element method for solving the Helmholtz equation in two dimensions with minimal pollution. Comput Methods Appl Mech Eng 1995;128(3):325-59.

[5] Melenk JM, Babuška I. Approximation with harmonic and generalized harmonic polynomials in the partition of unity method. Comput Assist Mech Eng Sci 1997;4:607-32.

[6] Strouboulis T, Copps K, Babuška I. The generalized finite element method: an example of its implementation and illustration of its performance. Int J Numer Methods Eng 2000;47:1401-17.

[7] Hughes T. Multiscale phenomena: Green's functions, the dirichlet-to-neumann formulation, subgrid scale models, bubbles and the origins of stabilized methods. Comput Methods Appl Mech Eng 1995;127(1):387-401.

[8] Franca LP, Farhat C, Macedo AP, Lesoinne M. Residual-free bubbles for the Helmholtz equation. Int J Numer Methods Eng 1997;40(21):4003-9.

[9] Dey S, Datta DK. A parallel hp-fem infrastructure for three-dimensional structural acoustics. Int J Numer Methods Eng 2006;68(5):583-603.

[10] Harari I, Magoulès F. Numerical investigations of stabilized finite element computations for acoustics. Wave Motion 2004;39(4):339-49.

[11] Demkowicz L. Computing with hp-adaptive finite elements: volume 1 one and two dimensional elliptic and maxwell problems, vol. 1. Chapman and Hall/ CRC; 2006.

[12] E. Trefftz, Ein gegenstuck zum ritzschen verfahren. In: Second international congress on applied mechanics; 1926. p. 131-7.

[13] Laghrouche O, Bettess P. Short wave modelling using special finite elements. J Comput Acoust 2000;8(1):189-210.

[14] Strouboulis T, Hidajat R. Partition of unity method for Helmholtz equation: $q$ convergence for plane-wave and wave-band local bases. Appl Math 2006:51:181-204.

[15] Cessenat O, Despres B. Application of an ultra weak variational formulation of elliptic pdes to the two-dimensional Helmholtz problem. SIAM J Numer Anal 1998;35:255-99.

[16] Huttunen T, Kaipio JP, Monk P. The perfectly matched layer for the ultra weak variational formulation of the 3D Helmholtz equation. Int J Numer Methods Eng 2004;61(7):1072-92.

[17] Gabard G, Gamallo P, Huttunen T. A comparison of wave-based discontinuous Galerkin, ultra-weak and least-square methods for wave problems. Int J Numer Methods Eng 2011;85(3):380-402. 
[18] Monk P, Wang D. A least-squares method for the Helmholtz equation. Comput Methods Appl Mech Eng 1999;175:121-36.

[19] Farhat C, Harari I, Franca L. The discontinuous enrichment method. Comput Methods Appl Mech Eng 2001;190:6455-79.

[20] Farhat C, Wiedemann-Goiran P, Tezaur R. A discontinuous Galerkin method with plane waves and lagrange multipliers for the solution of short wave exterior Helmholtz problems on unstructured meshes. Wave Motion 2004;39(4):307-17.

[21] Farhat C, Tezaur R, Toivanen J. A domain decomposition method for discontinuous Galerkin discretizations of Helmholtz problems with plane waves and lagrange multipliers. Int J Numer Methods Eng 2009;78(13): $1513-31$.

[22] Perrey-Debain E, Trevelyan J, Bettess P. Wave boundary elements: a theoretical overview presenting applications in scattering of short waves. Eng Anal Bound Elem 2004;28:131-41.

[23] Bériot H, Perrey-Debain E, Ben Tahar M, Vayssade C. Plane wave basis in Galerkin bem for bidimensional wave scattering. Eng Anal Bound Elem 2010;34(2):130-43.

[24] Desmet W, Sas P, Vandepitte D. An indirect Trefftz method for the steady-state dynamic analysis of coupled vibro-acoustic systems. Comput Assist Mech Eng Sci 2001;8(2-3):271-88.
[25] Van Hal B, Desmet W, Vandepitte D. Hybrid finite elementwave-based method for steady-state interior structural-acoustic problems. Comput Struct 2005;83(2):167-80.

[26] Van Genechten B, Atak O, Bergen B, Deckers E, Jonckheere S, Lee JS, et al. An efficient wave based method for solving Helmholtz problems in threedimensional bounded domains. Eng Anal Bound Elem 2012;36(1):63-75.

[27] Ladevèze P. A new computational approach for structure vibrations in the medium frequency range. CR Acad Sci Paris 1996;332(2b):849-56.

[28] Ladevèze P, Arnaud L. A new computational method for structural vibrations in the medium-frequency range. Comput Assist Mech Eng Sci 2000;7(2):219-26.

[29] Ladevèze P, Arnaud L, Rouch P, Blanzé C. The variational theory of complex rays for the calculation of medium-frequency vibrations. Eng Comput 2001;18(1/2):193-214.

[30] Ventsel E, Krauthammer T. Thin plates and shells: theory: analysis, and applications. CRC press; 2001.

[31] Kovalevsky L, Ladevèze $\mathrm{P}$, Riou $\mathrm{H}$, Bonnet $\mathrm{M}$. The variational theory of complex rays for three-dimensional Helmholtz problems. J Comput Acoust 2012;20:25. http://dx.doi.org/10.1142/S0218396X1250021X. Article ID: 1250021.

[32] Batoz J, Zheng C, Hammadi F. Formulation and evaluation of new triangular, quadrilateral, pentagonal and hexagonal discrete kirchhoff plate/shell elements. Int J Numer Methods Eng 2001;52(5-6):615-30. 